\title{
Dynamics of neutrophil-to-lymphocyte ratio predict outcomes of metastatic colorectal carcinoma patients treated by FOLFOX
}

\author{
Qian Liu ${ }^{1}$, Yanfeng $\mathrm{Xi}^{2}$, Guangzhao $\mathrm{He}^{3}$, Xiaoqian $\mathrm{Li}^{4}$, Feng Zhan ${ }^{1}$ \\ ${ }^{1}$ Department of Clinical Laboratory, Changzhou Cancer Hospital, Soochow University, Changzhou, China; ${ }^{2}$ Department of Surgical Oncology, \\ Changzhou Cancer Hospital, Soochow University, Changzhou, China; ${ }^{3}$ Department of Pharmacy, Changzhou Cancer Hospital, Soochow University, \\ Changzhou, China; ${ }^{4}$ Department of Oncology, Changzhou Cancer Hospital, Soochow University, Changzhou, China \\ Contributions: (I) Conception and design: Q Liu; (II) Administrative support: Y Xi; (III) Provision of study materials or patients: X Li; (IV) Collection \\ and assembly of data: F Zhan; (V) Data analysis and interpretation: G He; (VI) Manuscript writing: All authors; (VII) Final approval of manuscript: \\ All authors. \\ Correspondence to: Qian Liu. Department of Clinical Laboratory, Changzhou Cancer Hospital, Soochow University, 68 Red River Road, Changzhou \\ 213032, China. Email: liuqian_8686@aliyun.com.
}

Background: Peripheral blood cell count is the most common clinical laboratory test. Neutrophil-tolymphocyte ratio (NLR) as an economic marker has been reported in various cancer types. It is believed that NLR is associated with the prognosis and treatment outcomes of some cancers. Low baseline NLR has been reported as associated with better overall survival (OS) in advanced cancer patients. In this study, we aimed to determine whether the changes of NLR may predict the outcome of metastatic colorectal carcinoma (mCRC) patients treated with folinic acid, fluorouracil, and oxaliplatin (FOLFOX) combined with bevacizumab/ cetuximab.

Methods: The clinical data obtained from 128 mCRC patients between January 2014 and December 2018 were retrospectively analyzed. The NLR values of patients were calculated after 4 cycles of treatments. Kaplan-Meier analysis and Cox regression modeling were performed to assess the impact of NLR dynamics on OS and progression-free survival (PFS).

Results: Among the 128 participants, the optimum pre-treatment NLR cutoff value was 3. A total of $70(54.7 \%)$ participants had a pre-treatment of NLR lower than 3. The median of PFS was 9.1 months for NLR <3 compared with 6.1 months for pre-treatment NLR >3. A lower pre-treatment NLR was significantly associated with better PFS $(\mathrm{P}<0.001)$, but not associated with OS $(\mathrm{P}=0.064)$. A total of 94 (73.4\%) participants had a post-treatment NLR $<3$, which was associated with better PFS and OS $(\mathrm{P}=0.007)$. However, changes in NLR significantly affected PFS and OS. Decrease in post-treatment NLR was associated with longer PFS and OS. Patients with changes from low pre-treatment NLR to high posttreatment NLR had worse OS and PFS than that of NLR changes from high to low.

Conclusions: It is not the NLR but the changes of NLR that may predict the efficacy of FOLFOX treatment in mCRC patients.

Keywords: Metastatic colorectal carcinoma (mCRC); neutrophil-to-lymphocyte ratio (NLR); Folinic acid, fluorouracil, and oxaliplatin (FOLFOX); bevacizumab/cetuximab

Submitted Sep 30, 2021. Accepted for publication Dec 06, 2021.

doi: 10.21037 /jgo-21-716

View this article at: https://dx.doi.org/10.21037/jgo-21-716 


\section{Introduction}

The incidence of colorectal cancer (CRC) is increasing worldwide. In 2018, CRC ranked third in terms of incidence, and second in terms of mortality globally (1). Although great improvements in therapeutic strategies including chemotherapy and surgical treatments have been made, the mortality of CRC has remained high (2).

In the past decades, some novel treatments have been reported. Folinic acid, fluorouracil, and oxaliplatin (FOLFOX) combined with bevacizumab/cetuximab as the first-line chemotherapy has played an important role in metastatic colorectal carcinoma (mCRC) patients. However, FOLFOX might exert severe toxicity and side-effects to some mCRC patients due to patient heterogeneity. Therefore, predictive markers for the effect of FOLFOX on mCRC patients are needed before individualized treatment may progress.

Peripheral blood cell count is the most common clinical laboratory test. The count of neutrophils, lymphocytes, and monocytes are special markers for host immunity, and may reflect the condition of cytokine activation and some diseases. It is neutrophils not monocytes or macrophages were essential to deter tumor-infiltrating bacteria and dampen CRC promoting inflammation. Neutrophils represent an important component of the tumor microenvironment (3). Neutrophil specific IL$1 \mathrm{R}$ signaling controlled bacterial invasion and tumor elicited inflammation (4). It is believed that neutrophil-tolymphocyte ratio (NLR) (5-8), Monocyte-to-lymphocyte ratio (MLR) and Plate-to-lymphocyte ratio (PLR) (9-11) are associated with the prognosis and treatment outcomes of some cancers, include CRC. Previous studies have indicated that an elevated NLR before surgery or chemotherapy is associated with poor outcome in CRC patients $(12,13)$. NLR yielded a significant difference in five-year progression-free survival (PFS) (14). in lung metastasectomy for CRC patients. It shows that NLR is a strong predictor of outcome in mCRC patients undergoing pulmonary metastasectomy $(11,15)$. However the role of PLR in the prognosis of CRC patients is still controversial. Yang et al. (16) indicated that elevated PLR was significantly associated with poor PFS but not with OS. However, Jiang et al. (17) hold that PLR was not significantly associated with either PFS or OS. Many studies agreed that NLR was an independent prognostic factor not only for PFS but also for OS in mCRC patients treated by FOLFOX. But most researches evaluated the pre-treatment NLR.
Post-treatment NLR was rarely studied. Therefore, we evaluate pre-treatment NLR and post-treatment NLR simultaneously. We believe changes in the NLR may be a useful predictor for CRC patients' outcomes. In this study, we retrospectively investigated the relationship between the outcomes of FOLFOX treatment in mCRC patients and the change of NLR. This study might be helpful for developing a predictive marker regarding the efficacy of FOLFOX chemotherapy in mCRC patients. We present the following article in accordance with the STARD reporting checklist (available at https://dx.doi.org/10.21037/jgo-21-716).

\section{Methods}

\section{Patients and data collection}

In this study, we retrospectively enrolled all mCRC patients treated with FOLFOX at our hospital from January 2014 to December 2018. The inclusion criteria were as follows: stage IV CRC patients without previous chemotherapy history. Patients were excluded from the analysis if they had active infectious disease, anemia, hematological disorder, a treatment history of hypertension, cardiac failure, autoimmune disease, or a history of other malignancies.

The clinical data collected from electrical medical records included age, gender, date of diagnosis, initial stage, localization of the tumor, histological data, site of metastasis, and all subsequent treatments.

The complete blood count (CBC) was detected using a hematology analyzer ADVIA 2120i (Siemens Corporation, Munich, Germany). The laboratory data collected from CBC included white blood cell count, absolute neutrophil count, and absolute lymphocyte count. The NLR was defined as the absolute neutrophil count divided by the absolute lymphocyte count. Baseline NLR (within 3 days of the first chemotherapy) was defined as NLR1, and NLR measured after 8 weeks ( 4 cycles) of treatments was defined as NLR2.

\section{Treatment assessment}

Tumor response was assessed according to the response evaluation criteria in solid tumors (RECIST 1.1) regarding complete response (CR), partial response (PR), stable disease (SD), and progressive disease (PD). The primary endpoint was PFS, which was defined as the period time from the start date of chemotherapy to the first documentation of progression. Overall survival (OS) was 
Table 1 Clinicopathological features and NLR

\begin{tabular}{|c|c|c|c|c|c|c|c|}
\hline Characteristics & $N(\%),(n=128)$ & High NLR1 $(n=70)$ & Low NLR1 $(n=58)$ & P-value & High NLR2 $(n=34)$ & Low NLR2 $(n=94)$ & P-value \\
\hline Female & $30(23.4)$ & 14 & 16 & & 4 & 26 & \\
\hline Male & $98(76.6)$ & 56 & 42 & & 30 & 68 & \\
\hline \multicolumn{8}{|l|}{ Tumor location } \\
\hline Right colon & $28(21.9)$ & 16 & 12 & & 10 & 18 & \\
\hline Rectum & $60(46.9)$ & 36 & 24 & & 16 & 44 & \\
\hline \multicolumn{8}{|l|}{ Metastatic sites } \\
\hline Lung metastatic & $26(20.3)$ & 14 & 12 & & 6 & 20 & \\
\hline Other & $40(31.3)$ & 24 & 16 & & 12 & 28 & \\
\hline \multicolumn{8}{|l|}{ Number of metastatic sites } \\
\hline $0-2$ & $88(68.7)$ & 46 & 42 & & 22 & 66 & \\
\hline$>2$ & 40 (31.3) & 24 & 16 & & 12 & 28 & \\
\hline ORR & & $40 \%$ & $68.90 \%$ & $<0.001$ & $23.50 \%$ & $63.80 \%$ & $<0.001$ \\
\hline
\end{tabular}

NLR, neutrophil-to-lymphocyte ratio; ORR, objective response rate.

the secondary endpoint, and it was defined as the time from the date of first chemotherapy to death from any cause. The end of the follow-up period was December 2019. The objective response (OR) was defined as either $\mathrm{CR}$ or PR, and a non-response was defined as either SD or PD. Participants were also evaluated for adverse events according to the National Cancer Institute (NCI) Common Toxicity Criteria, version 3.0 (https://ctep.cancer.gov/ protocoldevelopment/electronic_applications/docs/ctcaev3. pdf). The study protocol was approved by the Ethics Committees of Changzhou Cancer Hospital. All procedures performed in this study involving human participants were in accordance with the Declaration of Helsinki (as revised in 2013). Individual consent for this retrospective analysis was waived.

\section{Statistical analysis}

The software SPSS version 22 (IBM Corp., Armonk, NY, USA) was used for statistical analysis. Continuous and categorical variables were compared using the MannWhitney $U$ test and $\chi^{2}$ test, respectively. Receiver operating characteristic (ROC) curve analysis was used to determine the cutoff values for the NLR, and Youden index (YI) was used to identify the optimal cutoff values for the NLR. All cases were divided into high and low NLR groups according to the cutoff value. Comparison between groups was evaluated using one-way analysis of variance (ANOVA) or unpaired Student's $t$-tests. Multiple comparison between the groups was performed using Student-Newman-Keuls method. Logistic regression analysis was conducted to evaluate the clinicopathological factors. Kaplan-Meier analysis was used to calculate the survival probability. Significant indicators for survival determined in univariate analysis were assessed via multivariate analysis using Cox's proportional hazards model. A P-value $<0.05$ was considered statistically significant.

\section{Results}

\section{Baseline participant characteristics}

Baseline participant characteristics are presented in the Table 1 . The median age was 59 years (range, 21 to 76 years). There were $30(23.4 \%)$ female and $98(76.6 \%)$ male 


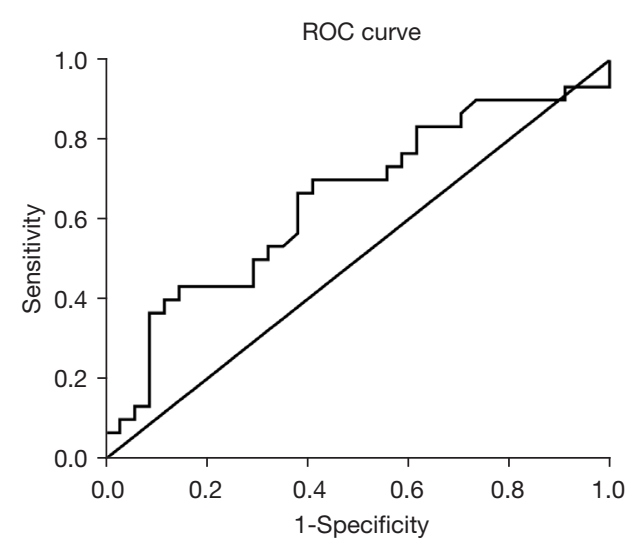

Figure 1 ROC curves analysis for NLR in mCRC patients. The AUC was 0.649 ( $\mathrm{P}=0.004$, 95\% CI: 0.552 to 0.749 ). ROC, receiver operating characteristic; NLR, neutrophil-to-lymphocyte ratio; mCRC, metastatic colorectal carcinoma; AUC, area under the curve; CI, confidence interval.

participants. A total of $40(31.2 \%)$ tumors were located in the left colon, $28(21.9 \%)$ were in the right colon, and $60(46.9 \%)$ were in the rectum. There were $26(20.3 \%)$ participants with only lung metastases, 48 (37.5\%) with only liver metastases, and 14 (10.9\%) participants had both lung and liver metastases. There were $40(31.3 \%)$ participants with more than 2 metastases.

\section{Prediction of chemotherapy efficacy based on the NLR}

The pre-treatment median NLR value of all participants was 3.15 and the median NLR of participants after 8 weeks (4 cycles) of treatment was 3.04. An ROC curve was constructed to estimate the optimal cutoff value of the pretreatment NLR for predicting clinical response (Figure 1). The area under the curve (AUC) was 0.649, and the optimal cutoff value was 3.0. Participants were then divided into 2 groups according to the pre-treatment NLR: low NLR1 (pre-treatment NLR <3.0, n=70, 54.7\%) and high NLR1 (pre-treatment NLR >3.0, n=58, 45.3\%). While the 2 groups had similar characteristics (Table 1), the low NLR1 group demonstrated a better overall response rate (ORR) $(68.9 \%$ vs. $40 \% ; \mathrm{P}<0.001)$. Participants were then categorized into the following groups: Low NLR2 group (those with NLR2 <3.0, n=94, 73.4\%) and High NLR2 group (those with NLR2 $>3.0, n=34,26.6 \%$ ). Participants with an NLR1 $<3.0$ had an ORR of $68.9 \%$. Participants with an NLR2 $<3.0$ after 8 weeks (4 cycles) of treatments were more likely to achieve an ORR (63.8\% vs. $23.5 \%$;
$\mathrm{P}<0.001 ;$ Table 1).

\section{Survival analysis of prognostic factors}

The median OS of all 128 participants was 25.1 months, and the median PFS was 8.5 months. The median PFS was 9.1 months $(95 \% \mathrm{CI})$ in the low NLR1 group and 6.1 months in the high NLR1 group $(\mathrm{P}<0.001)$. The median OS was 28.7 months (95\% CI) in the low NLR1 group and 21.9 months in the high NLR1 group ( $\mathrm{P}=0.064$; Figure 2). After 8weeks of treatment, participants with low NLR (low NLR2 group) had PFS of 8 months and OS of 23.9 month, compared to high NLR2 group for PFS of 4.2 months $(\mathrm{P}<0.001)$ and $\mathrm{OS}$ of 18.6 months $(\mathrm{P}=0.007$; Figure 3$)$. Therefore, the baseline NLR was significantly associated with PFS, but not associated with OS.

To investigate the relationship between NLR variation trend and prognosis of patients, we divided all participants into 4 groups: low-low group (NLR1 $<3.0$ and NLR2 $<3.0$ : 40 participants, $31.2 \%$ ), low-high group (NLR1 $<3.0$ and NLR2 >3.0: 18 participants, 14.1\%), high-low group (NLR1 $>3.0$ and NLR2 <3.0: 48 participants, 37.5\%), and highhigh group (NLR1 $<3.0$ and NLR2 <3.0: 22 participants, $17.2 \%)$. Patients with a persistently low NLR had the best PFS across the 4 groups. Patients with high NLR1 but low NLR2 had an improved PFS of 7 months (95\% CI), and those with a persistently high NLR had a PFS of 4 months (Figure 4). Although low baseline NLR could benefit more from the chemotherapy, NLR after 4 cycles was also important for PFS and OS.

Univariate analysis revealed that gender, age, tumor location, metastatic sites, and number of metastatic sites were not significantly associated with survival rate.

\section{Discussion}

The prognosis of tumor patients is closely related to their systemic immune status. Neutrophils can promote tumor invasion and metastasis by releasing elastase and cathepsin, which can hydrolyze antitumor factors (18). Neutrophil-derived leukotrienes can selectively amplify the subpopulations of cancer cells with high tumorigenic potential to help distant tissues to colonize. Lymphocyte is the main effector cell of immunity, and NLR may reflect the balance between pro-tumor inflammatory response and antitumor immune response $(12,19)$. Baseline NLR has been described as a prognostic indicator in various cancers $(20,21)$. In this study, we investigated whether NLR can 

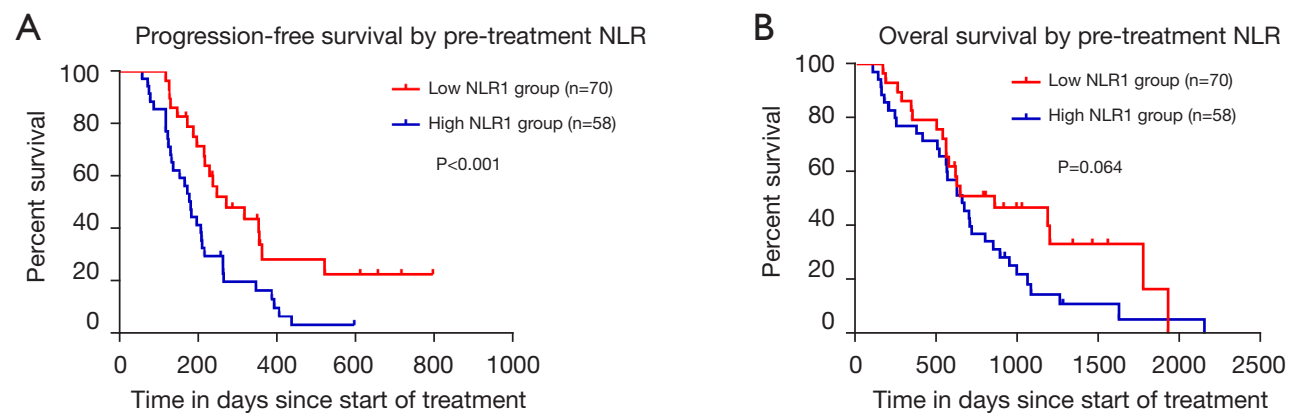

Figure 2 Kaplan-Meier curves of 128 mCRC patients determined by pre-treatment NLR. (A) PFS of 128 mCRC patients determined by pre-treatment NLR; (B) OS of 128 mCRC patients determined by pre-treatment NLR. PFS, progression-free survival; OS, overall survival; mCRC, metastatic colorectal cancer; NLR, neutrophil-to-lymphocyte ratio.
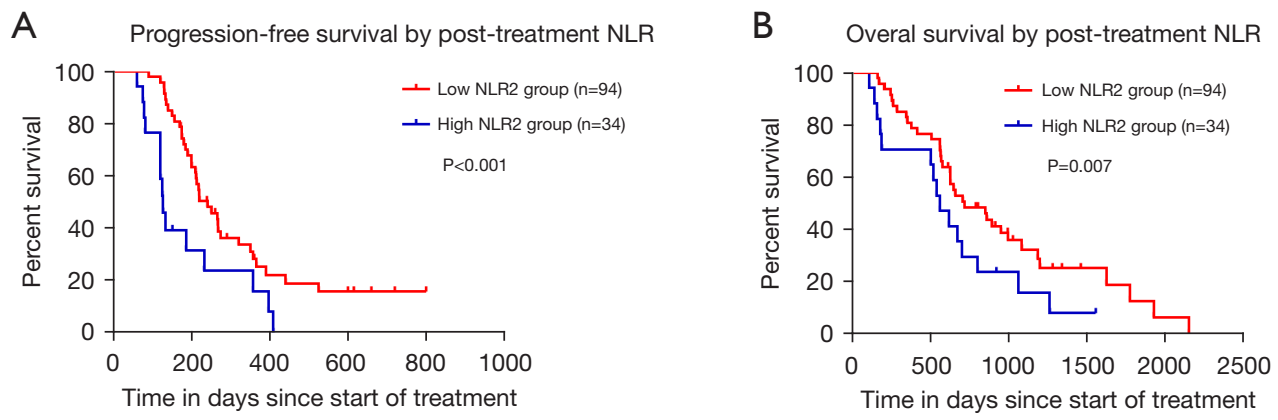

Figure 3 Kaplan-Meier curves of 128 mCRC patients determined by post-treatment NLR. (A) PFS of 128 mCRC patients determined by post-treatment NLR; (B) OS of 128 mCRC patients determined by post-treatment NLR. progression-free survival; OS, overall survival; mCRC, metastatic colorectal cancer; NLR, neutrophil-to-lymphocyte ratio.
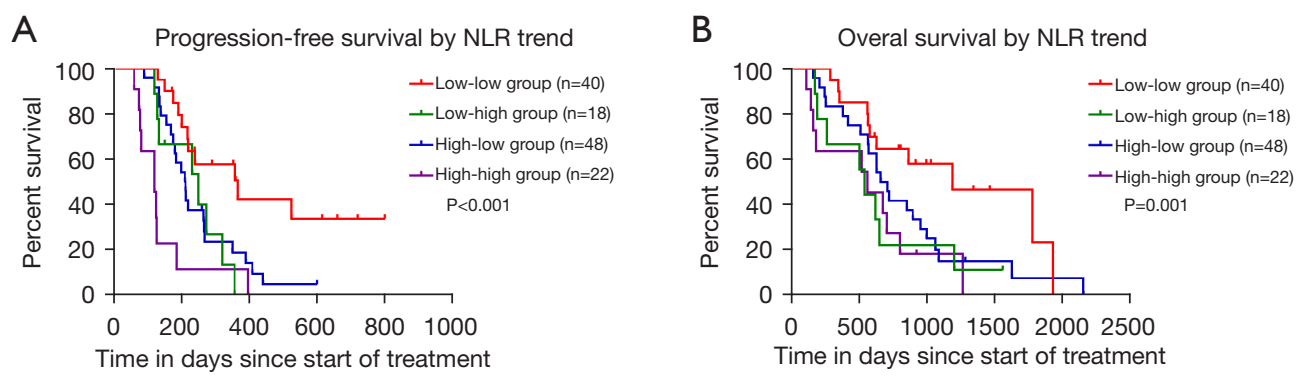

Figure 4 Kaplan-Meier curves for PFS of 128 mCRC patients according to the NLR trends before and after 4 cycles chemotherapy. (A) PFS of 128 mCRC patients according to the NLR trends before and after 4 cycles chemotherapy; (B) OS of $128 \mathrm{mCRC}$ patients according to the NLR trends before and after 4 cycles chemotherapy. PFS, progression-free survival; OS, overall survival; mCRC, metastatic colorectal cancer; NLR, neutrophil-to-lymphocyte ratio.

predict the therapeutic effect of FOLFOX in $128 \mathrm{mCRC}$ patients.

In this study, the cutoff value for baseline NLR was determined to be 3.0 according to the ROC curve.
This cutoff can effectively distinguish the efficacy of chemotherapy. The cutoff of NLR ranged from 2 to 5 in different diseases, race, and ages. Therefore, it is necessary to establish the NLR cutoff in different population 
demographics according to disease species and race with a large sample size in the future.

Baseline NLR has been reported to be associated with survival in cancer patients treated with both conventional chemotherapy and targeted treatments $(22,23)$. Baseline NLR was found to be correlated with OS in advanced cancer treated with programmed cell death protein 1/ programmed death ligand 1 (PD-1/PD-L1) inhibitors (24). However, our study showed that an elevated baseline NLR greater than the cutoff of 3 was associated with worse PFS, and that baseline NLR was not correlated with OS. This result may be explained by the limited sample size and specific metastatic CRC.

We then analyzed the NLR changes in mCRC patients from pre-treatment to 4 cycles of treatment with FOLFOX, to evaluate the significance of NLR as an independent biomarker in predicting clinical benefit in terms of PFS and OS. The Kaplan-Meier cumulative survival rates for OS and PFS demonstrated that decrease in NLR after 4 cycles of treatment with FOLFOX was associated with longer PFS and OS. Therefore, mCRC patients with reduced NLR after 4 cycles FOLFOX chemotherapy can benefit more from chemotherapy, regardless of their baseline NLR level. Interestingly, patients with changes in NLR from low baseline NLR to high post-treatment NLR had worse OS and PFS than those with NLR changes from high baseline to low post-treatment. According to the results mentioned above, it is not the post-treatment NLR but the changes in temporal profiles of NLR that are important.

Compared to a baseline NLR, a post-treatment NLR more objectively reflects the dynamic change in inflammatory responses of the body after FOLFOX treatment. Thus, NLR variation may directly predict the outcomes in mCRC patients undergoing FOLFOX chemotherapy. The fact that mCRC patients with an increased post-treatment NLR had higher progression rates suggests that FOLFOX treatment may have aggravated the inflammatory response which contributed to the tumor progression in these patients.

The NLR can be obtained from peripheral blood and act as an easy and cost-effective biomarker for survival in patients with FOLFOX chemotherapy. In CRC patients, whose life expectancy is relatively short, the efficacy of FOLFOX chemotherapy must be assessed early. Patients can benefit more from replacement of the treatment package early if FOLFOX chemotherapy is predicted as valid.

The small-sized, retrospective study design may be the most obvious limitation of this study. We selected mCRC patients treated with FOLFOX primarily. Patients who had previously undergone surgery or other chemotherapy were excluded. A large sample and Multicenter study should be added for verification later. However, the economic endowment of patients may affect their treatment choice, and thus influence the OS.

\section{Conclusions}

The NLR is a valuable predictor for the clinical efficacy of FOLFOX chemotherapy for mCRC patients. Identification of patients with elevated pre-chemotherapy or increased post chemotherapy NLRs will predict early recurrence and allow for timely interventions. The efficacy of chemotherapy can be also predicted by the variation trend of NLR after chemotherapy. Further studies conducted with larger prospective patient cohorts are needed to confirm the predictive role of NLR in FOLFOX chemotherapy.

\section{Acknowledgments}

Funding: None.

\section{Footnote}

Reporting Checklist: The authors have completed the STARD reporting checklist. Available at https://dx.doi. org/10.21037/jgo-21-716

Data Sharing Statement: Available at https://dx.doi. org/10.21037/jgo-21-716

Conflicts of Interest: All authors have completed the ICMJE uniform disclosure form (available at https://dx.doi. org/10.21037/jgo-21-716). The authors have no conflicts of interest to declare.

Ethical Statement: The authors are accountable for all aspects of the work in ensuring that questions related to the accuracy or integrity of any part of the work are appropriately investigated and resolved. The study protocol was approved by the Ethics Committees of Changzhou Cancer Hospital. All procedures performed in this study involving human participants were in accordance with the Declaration of Helsinki (as revised in 2013). Individual consent for this retrospective analysis was waived.

Open Access Statement: This is an Open Access article 
distributed in accordance with the Creative Commons Attribution-NonCommercial-NoDerivs 4.0 International License (CC BY-NC-ND 4.0), which permits the noncommercial replication and distribution of the article with the strict proviso that no changes or edits are made and the original work is properly cited (including links to both the formal publication through the relevant DOI and the license). See: https://creativecommons.org/licenses/by-nc-nd/4.0/.

\section{References}

1. Bray F, Ferlay J, Soerjomataram I, et al. Global cancer statistics 2018: GLOBOCAN estimates of incidence and mortality worldwide for 36 cancers in 185 countries. CA Cancer J Clin 2018;68:394-424.

2. Douillard JY, Oliner KS, Siena S, et al. PanitumumabFOLFOX4 treatment and RAS mutations in colorectal cancer. N Engl J Med 2013;369:1023-34.

3. Fridlender ZG, Sun J, Kim S, et al. Polarization of tumorassociated neutrophil phenotype by TGF-beta: "N1" versus "N2" TAN. Cancer Cell 2009;16:183-194.

4. Dmitrieva-Posocco O, Dzutsev A, Posocco DF, et al. Cell-Type-Specific Responses to Interleukin-1 Control Microbial Invasion and Tumor-Elicited Inflammation in Colorectal Cancer. Immunity 2019;50:166-180.e7.

5. Guo D, Han A, Jing W, et al. Preoperative to postoperative change in neutrophil-to-lymphocyte ratio predict survival in colorectal cancer patients. Future Oncol 2018;14:1187-96.

6. Hirahara N, Matsubara T, Mizota Y, et al. Prognostic value of preoperative inflammatory response biomarkers in patients with esophageal cancer who undergo a curative thoracoscopic esophagectomy. BMC Surg 2016;16:66.

7. Kang KH, Efird JT, Sharma N, et al. Prognostic potential of neutrophil-to-lymphocyte ratio and lymphocyte nadir in stage III non-small-cell lung cancer. Future Oncol 2017;13:1405-14.

8. Tangthongkum M, Tiyanuchit S, Kirtsreesakul V, et al. Platelet to lymphocyte ratio and red cell distribution width as prognostic factors for survival and recurrence in patients with oral cancer. Eur Arch Otorhinolaryngol 2017;274:3985-92.

9. Zhu JY, Liu CC, Wang L, et al. Peripheral blood lymphocyte-to-monocyte ratio as a prognostic factor in advanced epithelial ovarian cancer: A multicenter retrospective study. J Cancer 2017;8:737-43.

10. Guo YH, Sun HF, Zhang YB, et al. The clinical use of the platelet/lymphocyte ratio and lymphocyte/monocyte ratio as prognostic predictors in colorectal cancer: a metaanalysis. Oncotarget 2017;8:20011-24.

11. Wu Y, Li C, Zhao J, et al. Neutrophil-to-lymphocyte and platelet-to-lymphocyte ratios predict chemotherapy outcomes and prognosis in patients with colorectal cancer and synchronous liver metastasis. World J Surg Oncol 2016;14:289.

12. Kayadibi H, Sertoglu E, Uyanik M, et al. Neutrophillymphocyte ratio is useful for the prognosis of patients with hepatocellular carcinoma. World J Gastroenterol 2014;20:9631-2.

13. Nogueira-Costa G, Fernandes I, Gameiro R, et al. Prognostic utility of neutrophil-to-lymphocyte ratio in patients with metastatic colorectal cancer treated using different modalities. Curr Oncol 2020;27:237-43.

14. Londero F, Grossi W, Parise O, et al. The Impact of Preoperative Inflammatory Markers on the Prognosis of Patients Undergoing Surgical Resection of Pulmonary Oligometastases. J Clin Med 2020;9:3378.

15. Okazaki Y, Shibutani M, Wang EN, et al. Prognostic Significance of the Immunological Indices in Patients Who Underwent Complete Resection of Pulmonary Metastases of Colorectal Cancer. In Vivo 2021;35:1091-100.

16. Yang J, Guo X, Wang M, et al. Pre-treatment inflammatory indexes as predictors of survival and cetuximab efficacy in metastatic colorectal cancer patients with wild-type RAS. Sci Rep 2017;7:17166.

17. Jiang J, Ma T, Xi W, et al. Pre-treatment inflammatory biomarkers predict early treatment response and favorable survival in patients with metastatic colorectal cancer who underwent first line cetuximab plus chemotherapy. Cancer Manag Res 2019;11:8657-68.

18. El Rayes T, Catena R, Lee S, et al. Lung inflammation promotes metastasis through neutrophil proteasemediated degradation of Tsp-1. Proc Natl Acad Sci U S A 2015;112:16000-5.

19. Gao F, Li X, Geng M, et al. Pretreatment neutrophillymphocyte ratio: an independent predictor of survival in patients with hepatocellular carcinoma. Medicine (Baltimore) 2015;94:e639.

20. Chang Z, Zheng J, Ma Y, et al. The neutrophil-tolymphocyte ratio as a predictor for recurrence of colorectal liver metastases following radiofrequency ablation. Med Oncol 2014;31:855.

21. Zhang J, Zhang Y, Yv X, et al. Prognostic value of combined preoperative prognostic nutritional index and neutrophil to lymphocyte ratio in esophageal squamous cell carcinoma. Transl Cancer Res 2020;9:5117-27. 
22. Käsmann L, Bolm L, Schild SE, et al. Neutrophil-toLymphocyte Ratio Predicts Outcome in Limited Disease Small-cell Lung Cancer. Lung 2017;195:217-24.

23. Kuzman JA, Stenehjem DD, Merriman J, et al. Neutrophil-lymphocyte ratio as a predictive biomarker for response to high dose interleukin-2 in patients with renal cell carcinoma. BMC Urol 2017;17:1.

Cite this article as: Liu Q, Xi Y, He G, Li X, Zhan F. Dynamics of neutrophil-to-lymphocyte ratio predict outcomes of metastatic colorectal carcinoma patients treated by FOLFOX. J Gastrointest Oncol 2021;12(6):2846-2853. doi: 10.21037/jgo-21716
24. Moschetta M, Uccello M, Kasenda B, et al. Dynamics of Neutrophils-to-Lymphocyte Ratio Predict Outcomes of PD-1/PD-L1 Blockade. Biomed Res Int 2017;2017:1506824.

(English Language Editor: J. Jones) 